\title{
Impacto da pandemia COVID-19 no comportamento sedentário e nível de atividade física de professores da rede estadual de um município do Norte de Minas Gerais
}

Impact of the COVID-19 pandemic on sedentary behavior and physical activity level of state school

teachers in a municipality in the North of Minas Gerais

Impacto de la pandemia COVID-19 en el comportamiento sedentario y el nivel de actividad física

de docentes de escuelas estatales en un municipio del norte de Minas Gerais

Recebido: 20/08/2021 | Revisado: 28/08/2021 | Aceito: 03/09/2021 | Publicado: 05/09/2021

\author{
Ana Carolinny de Sousa \\ ORCID: https://orcid.org/0000-0001-8459-0658 \\ Faculdade Verde Norte, Brasil \\ E-mail: anacarolinyy10@gmail.com \\ Kênia Rayane Alves Miranda \\ ORCID: https://orcid.org/0000-0003-1303-0287 \\ Faculdade Verde Norte, Brasil \\ E-mail: keniarayane@outlook.com \\ Fernanda Muniz Vieira \\ ORCID: https://orcid.org/0000-0002-9051-0850 \\ Faculdade Verde Norte, Brasil \\ E-mail: fe1995muniz@hotmail.com \\ Alenice Aliane Fonseca \\ ORCID: https://orcid.org/0000-0003-4154-041X \\ Faculdade Verde Norte, Brasil \\ E-mail: alenicealiane@gmail.com
}

\begin{abstract}
Resumo
Diante do contexto de pandemia pela COVID-19, a prática do isolamento social tem causado alguns impactos na vida dos professores, relacionadas ao uso das tecnologias digitais, somadas à sua vida conjugal, materna e doméstica e tantas outras atribuições que lhes são conferidas. Adicionalmente, causando um aumento do sedentarismo e inatividade física. Assim, este estudo tem como objetivo avaliar o impacto da pandemia COVID-19 no comportamento sedentário e nível de atividade física de professores da rede estadual de ensino de Monte Azul-Minas Gerais. Foi realizado um estudo descritivo de corte transversal e caráter quantitativo. Os participantes responderam a questionários online através do Google Forms, enviado via @Whatsapp. Utilizou-se teste Qui-quadrado para avaliar associações entre o comportamento sedentário e o nível de atividade física antes e durante o período da pandemia. A amostra foi composta por 72 professores. O comportamento sedentário dos professores aumentou significativamente $(\mathrm{p}=0,000)$, durante o isolamento social. Enquanto a prática de atividade física apresentou um aumento, não significativo $(\mathrm{p}=0,182)$. Apesar do aumento, a prevalência de professores que não praticam atividade física suficiente é alta, variando de $63,9 \%$ antes e 55,6\% durante o isolamento social. Conclui-se que o isolamento social pela pandemia COVID-19 resultou em um aumento significativo do comportamento sedentário é uma alta prevalência de atividade física insuficiente entre professores da rede estadual de ensino. Desta forma, o estudo traz implicações práticas para que sejam pensadas formas de intervenção a essa população afim de incentivar a prática de atividade física e reduzir o tempo sentado durante períodos de distanciamento social.
\end{abstract}

Palavras-chave: COVID-19; Comportamento sedentário; Atividade física; Professores.

\begin{abstract}
In the face of the pandemic context by COVID-19, the practice of social isolation has caused some impacts on the lives of teachers, related to the use of digital technologies, added to their conjugal, maternal and domestic lives and so many other attributions that are given to them. Additionally, causing an increase in physical inactivity and physical inactivity. Thus, this study aims to assess the impact of the COVID-19 pandemic on the sedentary behavior and level of physical activity of teachers from the state school system in Monte Azul-Minas Gerais. A descriptive crosssectional and quantitative study was carried out. Participants answered online questionnaires through Google Forms, sent via@Whatsapp. A Chi-square test was used to assess associations between sedentary behavior and the level of physical activity before and during the pandemic period. The sample consisted of 72 teachers. Teachers' sedentary behavior increased significantly $(\mathrm{p}=0.000)$ during social isolation. While the practice of physical activity showed an increase, it was not significant $(\mathrm{p}=0.182)$. Despite the increase, the prevalence of teachers who do not practice
\end{abstract}


enough physical activity is high, ranging from $63.9 \%$ before and $55.6 \%$ during social isolation. It is concluded that social isolation by the pandemic COVID-19 resulted in a significant increase in sedentary behavior is a high prevalence of insufficient physical activity among teachers in the state school system. In this way, the study has practical implications for thinking about forms of intervention for this population in order to encourage the practice of physical activity and reduce sitting time during periods of social distance.

Keywords: COVID-19; Sedentary behavior; Physical activity; Teachers.

\section{Resumen}

Dado el contexto de la pandemia por COVID-19, la práctica del aislamiento social ha provocado algunos impactos en la vida de los docentes, relacionados con el uso de tecnologías digitales, sumado a su vida conyugal, materna y doméstica y tantas otras atribuciones que se le confieren ellos. Además, provoca un aumento de la inactividad física y la inactividad física. Así, este estudio tiene como objetivo evaluar el impacto de la pandemia COVID-19 en el comportamiento sedentario y el nivel de actividad física de los docentes del sistema educativo estatal de Monte AzulMinas Gerais. Se realizó un estudio descriptivo, transversal y cuantitativo. Los participantes completaron cuestionarios en línea a través de Google Forms, enviados a través de @Whatsapp. La prueba de Chi-cuadrado se utilizó para evaluar las asociaciones entre el comportamiento sedentario y el nivel de actividad física antes y durante el período pandémico. La muestra estuvo formada por 72 profesores. El comportamiento sedentario de los profesores aumentó significativamente $(\mathrm{p}=0,000)$ durante el aislamiento social. Si bien la práctica de actividad física mostró un aumento, no fue significativo $(\mathrm{p}=0,182)$. A pesar del aumento, la prevalencia de docentes que no practican suficiente actividad física es alta, oscilando entre el 63,9\% antes y el 55,6\% durante el aislamiento social. Se concluye que el aislamiento social provocado por la pandemia COVID-19 resultó en un aumento significativo de la conducta sedentaria y una alta prevalencia de actividad física insuficiente entre los docentes de las escuelas públicas. De esta forma, el estudio tiene implicaciones prácticas para que se puedan pensar formas de intervención en esta población con el fin de incentivar la práctica de actividad física y reducir el tiempo sentado en períodos de distanciamiento social.

Palabras clave: COVID-19; Comportamiento sedentario; Actividad física; Maestros.

\section{Introdução}

A doença do coronavírus COVID-19, é uma síndrome respiratória aguda grave causada pelo coronavírus 2 da SARS (SARS-CoV-2) (Wu et al., 2020). O SARS-CoV-2 é caracterizado como um novo subtipo de síndrome respiratória aguda grave (SARS-CoV) humana com alta capacidade de transmissão e indução de quadros de infecção respiratória severa (Croda \& Garcia, 2020). O popularmente COVID-19 de forma rápida e incontrolável se disseminou assinalando uma pandemia mundial (World Health Organization, 2020).

Dentre os setores impactados pela pandemia está a educação, que de forma efetiva alterou a rotina das escolas em todo o mundo. Uma vez que, para conter a disseminação do COVID-19, a maioria das instituições de ensino seja, do ensino básico, profissionalizante ou superior, foram fechadas para evitar as aglomerações de estudantes e professores nas salas de aula e nos outros espaços acadêmicos, retardando a propagação da infecção (Daniel, 2020).

Houve a transferência do ensino presencial para atividades educativas emergenciais (Sampaio, 2020). Os recursos mais utilizados são o envio de materiais digitais pelo professor aos alunos, envio de orientações via redes sociais para que os estudantes acessem em casa com apoio de livros didáticos, e disponibilização de videoaulas gravadas pelos professores e enviadas aos estudantes por redes sociais para que eles assistam em seus aparelhos.

Em Minas Gerais (2020), as atividades pedagógicas não presenciais vêm acontecendo por meios digitais ou por meio do Plano de Estudos Tutorados (PET), que consiste em um instrumento de aprendizagem baseado em atividades autoinstrucionais a ser disponibilizado a todos os estudantes, por meio de recursos das Tecnologias de Informação e Comunicação (TICs) compatíveis com as condições de acesso dos estudantes e, apenas excepcionalmente, por meio de materiais impressos com orientações pedagógicas distribuído aos alunos e seus pais ou responsáveis.

Dessa forma, diante das medidas adotadas surgem desafios para os professores relacionados ao aprendizado do manuseio dos TICs, acompanhamento e correção do PET, além de estimular os discentes na construção e busca do conhecimento e assim garantir a finalização do ano letivo, somadas à sua vida conjugal, materna e doméstica e tantas outras 
atribuições que lhes são conferidas, causando um aumento do sedentarismo e inatividade física (Ammar et al., 2020) além de repercutir na saúde mental.

Durante o isolamento domiciliar a população tende a adotar uma rotina sedentária (Bezerra, Silva, Soares \& Silva, 2020), favorecendo o aumento no ganho de peso corporal e surgimento de comorbidades (Ferreira, Irigoyen, ConsolimColombo, Saraiva \& De Angelis, 2020). O comportamento sedentário é definido como atividades caracterizadas por baixo gasto energético, não excedendo 1,5 equivalentes metabólicos que incluem comportamentos específicos de sentar, reclinar ou deitar, para ler, estudar, assistir televisão, usar o computador, entre outras, excetuando-se as horas sono (Tremblay et al., 2017).

Neste contexto, é necessário investigar e incentivar a manutenção de uma rotina de vida fisicamente ativa como uma medida preventiva para a saúde durante esse período de enfrentamento contra a disseminação do vírus. Reduzindo o comportamento sedentário, ou seja, o tempo que permanecemos sentados, deitados ou reclinados durante o dia (Pitanga, Beck \& Pitanga, 2020), uma vez que, professores atuando em home office tendem a permanecerem mais tempo sentados executando suas atividades laborais. Com isso, o objetivo deste estudo foi analisar o impacto da pandemia COVID-19 no nível de atividade física e comportamento sedentário de professores da rede estadual do município de Monte Azul-Minas Gerais.

\section{Metodologia}

\section{Caracterização do estudo e aspectos éticos}

Trata-se de um estudo transversal de caráter quantitativo (Prodanov \& Freitas, 2013), realizado na cidade de Monte Azul-Minas Gerais. Este estudo foi aprovado pelo comitê de ética em pesquisa da Universidade Estadual de Montes Claros, sob parecer número 4.436 .467$.

\section{Procedimentos}

Atualmente o município conta com 07 escolas estaduais, sendo 06 urbanas e 01 rural. As escolas participantes foram selecionadas mediante sorteio aleatório simples, onde foram selecionadas 04 escolas para participação no estudo. Inicialmente, foi enviada a Secretaria Estadual de Educação, uma carta de apresentação do estudo solicitando autorização para realização da pesquisa. Feito isso, a direção das escolas sorteadas foi procurada a fim de requerer uma autorização para realização da pesquisa.

A seleção dos professores participantes foi realizada de forma não-probabilista, onde aqueles que atenderam aos critérios de seleção foram convidados a participarem do estudo. Os critérios de inclusão foram: ser professor de uma das escolas participantes, ter acesso à internet, e aceitar a participar do estudo de forma voluntária. Foram excluídos todos aqueles professores que estavam afastados ou com algum tipo de licença.

Dadas as restrições impostas pela pandemia pelo COVID-19, os participantes responderam a questionários usando um software de pesquisa online (Survey) através do Google Forms. Um link para a pesquisa eletrônica foi enviado aos professores via @ Whatsapp através do grupo dos professores das escolas.

Todos os entrevistados forneceram consentimento informado antes de acessar os questionários, tendo a garantia de que todos os dados seriam usados apenas para fins de pesquisa. As respostas dos participantes são anônimas e confidenciais, além disso, os participantes poderiam interromper a participação no estudo e sair do questionário em qualquer estágio antes do processo de envio, onde suas respostas não seriam salvas. Ao completar a pesquisa, os participantes reconheceram seu consentimento voluntário em participar do estudo. 


\section{Instrumentos}

Foram coletadas informações referentes aos dados sociodemográficos, prática de atividade física e comportamento sedentário. As questões referentes ao perfil sociodemográfico dos participantes abordaram as variáveis de idade, sexo, raça, estado conjugal, número de filhos, escolaridade, quantas pessoas moram na casa, em qual nível de ensino atua, tempo de docência, satisfação com o trabalho, tempo dedicado ao trabalho por dia.

Para avaliar o nível de atividade física foram questionados a prática de atividade física antes e durante o isolamento social: antes do isolamento socia, você praticava algum tipo de atividade física? (sim, não), quanto tempo você gastava realizando atividade física por dia?, quantas vezes na semana?; Durante o isolamento social, você tem praticado algum tipo de atividade física ( $\operatorname{sim}$, não), quanto tempo você gasta realizando atividade física por dia?, quantas vezes na semana?; Em relação a pratica atual de atividade física como você considera a mudança desde o início do isolamento (prático mais, prático a mesma coisa, prático menos).

As informações referentes ao comportamento sedentário foram questionadas antes e durante o isolamento social como: o tempo total gasto sentado, deitado e assistindo televisão durante um dia (horas). E quais as atividades tem realizado no dia a dia (assistir filmes/séries; ler, assistir televisão, fazer artesanato ou qualquer atividade artística; navegar ou compartilhar conteúdos em redes sociais, conversar com alguém (cara a cara ou via telefone, mensagens instantâneas, videochamadas ...), outras atividades de lazer.

Foi feito um cálculo de média ponderada da seguinte forma: tempo dos dias de semana multiplicado por 5, somado ao tempo dos dias de fim de semana multiplicado por 2, dividindo esse resultado por 7, para se obter o número médio de horas por dia dispendidos na posição sentada, como proposto no estudo de Rocha, Goldbaum, César e Stopa (2019).

\section{Análise dos dados}

Para análise dos dados, utilizou-se o programa estatístico SPSS (Statistical Package for the Social Sciences), versão 25. Inicialmente, foi realizada uma análise descritiva exploratória dos dados, com distribuição de frequências das variáveis do estudo. Em seguida foi realizado o teste qui-quadrado, sendo adotado o nível de significância de 5\% $(\mathrm{p}<0,05)$.

\section{Resultados}

A amostra foi composta por 72 professores, com média de idade de 42,65 (DP $\pm 8,05)$ anos. Destes a maioria era do sexo feminino (75\%), de cor parda $(77,8 \%)$, com companheiro $(61,1 \%)$, com apenas o ensino superior completo $(52,8 \%)$, atuantes no ensino fundamental (58,3\%), e médio (41,7\%), a mais de 10 anos (66,7\%). Mesmo diante do quadro pandêmico e aulas remotas, os professores estão satisfeitos com o trabalho $(66,7 \%)$, 50\% trabalham até quatro horas por dia e 50\%, mais que cinco horas, e relatam que a carga de trabalho aumentou com a pandemia $(68,1 \%)$. 
Tabela 1: Caracterização dos professores.

\begin{tabular}{|c|c|c|}
\hline \multicolumn{2}{|c|}{ Variáveis } & \multirow{2}{*}{$\frac{\mathbf{n}(\%)}{17(23,6)}$} \\
\hline Soyo & Masculino & \\
\hline Sexo & Feminino & $54(75,0)$ \\
\hline \multirow{2}{*}{ Cor } & Branca & $16(22,2)$ \\
\hline & Parda & $56(77,8)$ \\
\hline \multirow{2}{*}{ Estado conjugal } & Com companheiro(a) & $44(61,1)$ \\
\hline & Sem companheiro(a) & $28(38,9)$ \\
\hline \multirow{3}{*}{ Escolaridade } & Ensino Superior & $38(52,8)$ \\
\hline & Especialização & $19(26,4)$ \\
\hline & Mestrado-Doutorado & $14(19,4)$ \\
\hline \multirow{2}{*}{ Nível de ensino que atua } & Ensino Fundamental & $42(58,3)$ \\
\hline & Ensino Médio & $30(41,7)$ \\
\hline \multirow{2}{*}{ Tempo de docência } & $<10$ anos & $23(31,9)$ \\
\hline & $>10$ anos & $48(66,7)$ \\
\hline \multirow{3}{*}{ Satisfação no trabalho } & Satisfeito & $48(66,7)$ \\
\hline & Indiferente & $10(13,9)$ \\
\hline & Insatisfeito & $14(19,4)$ \\
\hline \multirow{2}{*}{ Tempo realizando atividades } & Até 4 horas por dia & $36(50)$ \\
\hline & 5 horas ou mais & $36(50)$ \\
\hline \multirow{3}{*}{ Carga de trabalho } & Aumentou & $49(68,1)$ \\
\hline & Permaneceu a mesma & $16(22,2)$ \\
\hline & Diminuiu & $07(09,7)$ \\
\hline
\end{tabular}

Fonte: Autores (2021).

A Tabela 2 apresenta o comportamento sedentário e a prática de atividade física dos professores antes e durante o isolamento social pela pandemia COVID-19. Pode-se observar que o comportamento sedentário aumentou significativamente $(\mathrm{p}=0,000)$, durante o isolamento social. Enquanto a prática de atividade física apresentou um aumento, não significativo $(\mathrm{p}=0,182)$. Apesar do aumento, a prevalência de professores que não praticam atividade física suficiente é alta, variando de $63,9 \%$ antes e $55,6 \%$ durante o isolamento social.

Tabela 2: Comportamento sedentário e prática de atividade física dos professores antes e durante o isolamento social pela pandemia COVID-19.

\begin{tabular}{|c|c|c|c|c|}
\hline & & \multicolumn{2}{|c|}{ Isolamento Social } & \multirow{3}{*}{ p-valor } \\
\hline & & Antes & Durante & \\
\hline & & n (\%) & n (\%) & \\
\hline \multirow{3}{*}{ Comportamento Sedentário } & Tempo Médio (DP) & $237,16(142,78)$ & $396,76(245,60)$ & \multirow[b]{3}{*}{$0,000^{*}$} \\
\hline & $<180 \mathrm{~min} /$ dia & $30(41,7)$ & $07(09,7)$ & \\
\hline & $>180 \mathrm{~min} / \mathrm{dia}$ & $42(58,3)$ & $65(90,3)$ & \\
\hline \multirow{3}{*}{ Atividade Física } & Tempo Médio (DP) & $198,14(171,42)$ & $214,14(121,42)$ & \\
\hline & $>150 \mathrm{~min} / \mathrm{semana}$ & $26(36,1)$ & $32(44,4)$ & \multirow{2}{*}{0,182} \\
\hline & $<150 \mathrm{~min} / \mathrm{semana}$ & $45(63,9)$ & $40(55,6)$ & \\
\hline
\end{tabular}

* Valor de p do teste de qui-quadrado de Pearson. Fonte: Autores (2021). 


\section{Discussão}

Até onde sabemos, este é o primeiro estudo que investigou os comportamentos sedentários e padrões de atividade física de professores antes e durante a pandemia de COVID-19. Descobrimos que 58,3\% dos professores participantes tinham um comportamento sedentário elevado antes da pandemia (ou seja, tempo gasto em atividades sedentárias >180 min/semana), e este número subiu para 90,3\% durante o isolamento social pela COVID-19. É possível que o aumento do número de professores com comportamento sedentário elevado se deu pelas atividades laborais exercidas durante o isolamento social, onde as atividades laborais são exercidas em casa, com uma maior demanda de tempo sentado em frente a um computador. Quanto ao nível de atividade física, a prevalência de professores que atingiram 150 minutos por semana de atividade física antes da pandemia passou de $36,1 \%$, para 44,4\%, entretanto, não apresentou diferença significativa $(\mathrm{p}=0,182)$.

A comparação dos achados com a literatura apresenta um desafio perante a especificidade do enfrentamento da pandemia e a ausência de outros estudos com essa população. No entanto, em um estudo realizado por Botero et al. (2021) com adultos brasileiros, indicam que o isolamento social imposto pela pandemia de COVID-19 levou a uma diminuição do nível de atividade física e ao aumento do comportamento sedentário, onde 82,3\% dos participantes aumentaram o tempo sentado devido à COVID-19. Já no estudo de Malta et al. (2020), também com adultos brasileiros, a prevalência de atividade física suficiente passou de $30,1 \%$ a $12,0 \%$ durante o isolamento.

Os achados deste estudo são preocupantes, já que a inatividade física e o maior tempo de permanência sentado são preditores independentes de mortalidade por doenças cardiovasculares (Ekelund et al., 2019). Segundo Couto, Couto e Cruz (2020) grande parte da rotina tem sido afetada resultando em um impacto nas atividades que eram realizadas pelas pessoas, um exemplo disso, é o aumento de pessoas obesas.

Como observado na Tabela 1 grande parte dos professores analisados são mulheres (75\%), casadas ou com companheiros $(61 \%)$, esse pode ser considerado um dos principais contextos dentro do mundo educativo, uma boa parte dos professores em atividade no Brasil são mulheres (Inep, 2004). Essas devido a algumas particularidades não realizam atividades físicas de forma rotineira, devido suas atividades de vida diárias. No que se refere a prática de atividade física verifica-se que existe um nivelamento (50\% para cada) dos professores que realizam atividades físicas e dos que não realizam, sendo observado dessa forma que algumas pessoas consideram a rotina de exercícios uma forma de estabilizar o seu corpo ou mente em uma atividade profissional que exige muito.

Neste contexto, é perceptível que os profissionais de educação precisaram conciliar a nova rotina de ensino com os exercícios ou atividades físicas, algo que concedeu aos mesmos uma visão diferenciada sobre como realizar as atividades físicas dentro do ambiente doméstico (Pitanga, Beck \& Pitanga, 2017). Estes achados podem ser resultantes da disseminação de rotinas físicas pelas redes sociais, onde os exercícios podem ser realizados dentro de casa. Segundo Oliveira Neto et al. (2020) a busca no Google pelo termo "treinamento em casa" tem crescido gradativamente, confirmando a relevância da manutenção de uma rotina de exercícios físicos durante o período de isolamento social.

Um dos pontos fundamentais observados consiste na valorização do conhecimento de que a prática de exercícios pode minimizar os impactos do sedentarismo. Como evidenciado por Ammar et al. (2020) as práticas de esportes ou atividades físicas foram um dos principais recursos usados ao longo do isolamento social, como uma forma de proporcionar os indivíduos uma fuga de uma limitação a grande prazo. Mesmo com as limitações físicas os indivíduos foram motivados a realizar algumas atividades em suas casas, alcançando uma certa minimização dos impactos físicos promovidos pela não interação com os meios externos, minimizando os riscos ou o desenvolvimento de doenças.

Mesmo com todos os incentivos promovidos e aplicados pelas mídias sociais, pode-se verificar que os indivíduos passaram a realizar poucas atividades físicas ao longo do isolamento social, algo que pode ser relacionado com a dificuldade em manter ou desenvolver uma rotina ativa em suas residências (Botero et al., 2021). O número de professores que 
aumentaram o tempo sentado e praticavam atividades físicas insuficientes durante o isolamento social ressaltam a necessidade de interação existente entre os indivíduos e as práticas de exercícios físicos, assim como consolidam a importância de uma rotina para que os mesmos consigam realizar atividades físicas.

Esses achados apontam para a necessidade de implementar medidas preventivas de saúde pública que incentivem a prática de atividades físicas durante a pandemia, diminuindo o comportamento sedentário. Este estudo apresenta algumas limitações, como seu desenho transversal e coleta de dados on-line, uma vez que são suscetíveis a vieses de informações, sobretudo quando não há indicadores diretos de atividade física e do comportamento sedentário. No entanto, durante o período de pandemia, as coletas on-line têm se mostrado uma alternativa para a realização de novos estudos durante a pandemia de COVID-19 (Schmidt, Palazzi \& Piccinini, 2020).

\section{Conclusão}

Conclui-se que o isolamento social pela pandemia COVID-19 resultou em um aumento significativo do comportamento sedentário é uma alta prevalência de atividade física insuficiente entre professores da rede estadual de ensino. Desta forma, o estudo traz implicações práticas para que sejam pensadas formas de intervenção a essa população afim de incentivar a prática de atividade física e reduzir o tempo sentado durante períodos de distanciamento social.

\section{Referências}

Ammar, A., Brach, M., Trabelsi, K., Chtourou, H., Boukhris, O., Masmoudi, L., Bouaziz, B., Bentlage, E., How, D., Ahmed, M., Müller, P., Müller, N., Aloui, A., Hammouda, O., Paineiras-Domingos, L. L., Braakman-Jansen, A., Wrede, C., Bastoni, S., Pernambuco, C. S., Mataruna, L., Taheri, M., Irandoust, K., Khacharem, A., Bragazzi, N. L., Chamari, K., Glenn, J. M., Bott, N. T., Gargouri, F., Chaari, L., Batatia, H., Ali, G. M., Abdelkarim, O., Jarraya, M., Abed, K. E., Souissi, N., Van Gemert-Pijnen, L., Riemann, B. L., Riemann, L., Moalla, W., Gómez-Raja, J., Epstein, M., Sanderman, R., Schulz, S. V., Jerg, A., AlHorani, R., Mansi, T., Jmail, M., Barbosa, F., Ferreira-Santos, F., Šimunič, B., Pišot, R., Gaggioli, A., Bailey, S. J., Steinacker, J. M., Driss, T. \& Hoekelmann, A. (2020). Effects of COVID-19 Home Confinement on Eating Behaviour and Physical Activity: Results of the ECLB-COVID-19 International Online Survey. Nutrients, 12(6),1583. https://doi.org/10.3390/nu12061583.

Bezerra, A. C. V., Silva, C. E. M., Soares, F. R. G. \& Silva, J. A. M. (2020). Fatores associados ao comportamento da população durante o isolamento social na pandemia de COVID-19. Ciência \& Saúde Coletiva, 25(1), 2411-2421. https://doi.org/10.1590/1413-81232020256.1.10792020

Botero, J. P., Farah, B. Q., Correia, M. A., Lofrano-Prado, M. C., Cucato, G. G., Shumate, G., Ritti-Dias, R. M. \& Prado, W. L. do. (2021). Impacto da permanência em casa e do isolamento social, em função da COVID-19, sobre o nível de atividade física e o comportamento sedentário em adultos brasileiros. einstein (São Paulo), 19:eAE6156. https://doi.org/10.31744/einstein_journal/2021_AE6156

Daniel, S. J. (2020). "Education and the COVID-19 pandemic.” Prospects. 20, 1-6. https://doi.org/10.1007/s11125-020-09464-3

Couto, E. S., Couto, E. S. \& Cruz, I. M. P. (2020) \#FIQUEEMCASA: Educação na Pandemia da COVID-19. Interfaces Científicas - Educação, 8(3), $200-217$. https://doi.org/10.17564/2316-3828.2020v8n3p200-217

Croda, J. H. R. \& Garcia, L. P. (2020). Immediate Health Surveillance Response to COVID-19 Epidemic. Epidemiologia e Serviços de Saúde, 29(1), e2020002. https://doi.org/10.5123/S1679-49742020000100021

Ekelund, U., Tarp, J., Steene-Johannessen, J., Hansen, B. H., Jefferis, B., Fagerland, M. W., Whincup, P., Diaz, K. M., Hooker, S. P, Chernofsky, A., Larson, M. G., Spartano, N., Vasan, R. S., Dohrn, I. M., Hagströmer, M., Edwardson. C., Yates, T., Shiroma, E., Anderssen, S. A. \& Lee, I. M. (2019). Dose-response associations between accelerometry measured physical activity and sedentary time and all-cause mortality: systematic review and harmonised meta-analysis. BMJ, 21, 366:4570. https://doi.org/10.1136/bmj.14570.

Ferreira, M. J., Irigoyen, M. C., Consolim-Colombo, F., Saraiva, J. F. K. \& De Angelis K. (2020). Vida Fisicamente Ativa como Medida de Enfrentamento ao COVID-19. Arquivos Brasileiros de Cardiologia, 114(4), 601-602. https://doi.org/10.36660/abc.20200235

Instituto Nacional de Estudos e Pesquisas Educacionais Anísio Teixeira (2004). Estatísticas dos professores no Brasil. (2a ed.), Inep, (2th ed., pp. 46)

Malta, D. C., Szwarcwald, C. L., Barros, M. B. de A., Gomes, C. S., Machado, Í. E., Souza Júnior, P. R. B. de, Romero, D. E., Lima, M. G., Damacena, G. N., Pina, M. de F., Freitas, M. I. de F., Werneck, A. O., da Silva, D. R. P., Azevedo, L. O. \& Gracie, R. (2020). A pandemia da COVID-19 e as mudanças no estilo de vida dos brasileiros adultos: um estudo transversal, 2020. Epidemiologia e Serviços de Saúde, 29(4). http://dx.doi.org/10.1590/s1679497420200004000026

Minas Gerais (Estado). Nota Jurídica PROEDUC/CREDCAS nº 2/2020. Apontamentos e sugestões de atuação às Promotorias de Justiça no acompanhamento da reorganização dos calendários escolares e da oferta de ensino não presencial por escolas da educação básica durante a suspensão das aulas presenciais em decorrência da pandemia da COVID-19. De http://www2.educacao.mg.gov.br/component/gmg/page/17164-boletim-de-legislacoes-e-normas-n-02-junho-2020

Muscogiuri, G., Barrea, L., Savastano, S. \& Colao, A. (2020). Nutritional recommendations for COVID-19 quarantine. European Journal of Clinical Nutrition, 74(6), 850-851. http://dx.doi.org/10.1038/s41430-020-0635-2 
Oliveira Neto, L., Elsangedy, H. M., Tavares, V. D. de O., Teixeira, C. V. L. S., Behm, D. G. \& Silva-Grigoletto, M. E. Da. (2020). \#TreineEmCasa Treinamento físico em casa durante a pandemia do COVID-19 (SARS CoV-2): abordagem fisiológica e comportamental. Revista Brasileira de Fisiologia do Exercício, 19(2), 9-19.

Pedrosa, N. L. \& Albuquerque, N. L. S. (2020). Analise Espacial dos Casos de COVID-19 e leitos de terapia intensiva no estado do Ceara, Brasil. Ciência \& Saúde Coletiva, 25(1),2461-8. https://doi.org/10.1590/1413-81232020256.1.10952020

Prodanov, C. C., \& Freitas, E. C. (2013). Metodologia do trabalho científico: métodos e técnicas da pesquisa e do trabalho acadêmico- $2^{\text {a }}$ Edição. Editora Feevale.

Pitanga, F. J. G., Beck, C. C. \& Pitanga, C. P. S. (2020). Atividade Física e Redução do Comportamento Sedentário durante a Pandemia do Coronavírus. Revista Brasileira De Atividade Física \& Saúde, 25, 1-4. https://doi.org/10.12820/rbafs.25e0114

Rocha, B. M. C., Goldbaum, M., César, C. L. G. \& Stopa, S. R. (2019). Comportamento sedentário na cidade de São Paulo: ISA-Capital 2015. Revista Brasileira de Epidemiologia. 22, e190050. https://doi.org/10.1590/1980-549720190050

Sampaio, R. M. (2020). Práticas de ensino e letramentos em tempos de pandemia da COVID -19. Research, Society and Development, 9(7), 1-16, e519974430. http://dx.doi.org/10.33448/rsd-v9i7.4430

Schmidt, B., Palazzi, A., \& Piccinini, C. A. (2020). Entrevistas online: Potencialidades e desafios para coleta de dados no contexto da pandemia de COVID19. REFACS, 8(4), 960-966. http://dx.doi.org/10.18554/refacs.v8i4.4877

Singhal, T. (2020). A Review of Coronavirus Disease-2019 (COVID-19). In Indian J Pediatr, 87(4), 281-286. http://dx.doi.org/10.1007/s12098-020-03263-6.

Tremblay, M. S., Aubert, S., Barnes, J. D., Saunders, T. J., Carson, V., Latimer-Cheung, A. E., Chastin, S. F. M., Altenburg, T. M., Chinapaw, M. J. M. \& SBRN Terminology Consensus Project Participants. (2017). Sedentary Behavior Research Network (SBRN) terminology consensus project process and outcome. Int J Behav Nutr Phys. 14(1):75. http://dx.doi.org/10.1186/s12966-017-0525-8.

Vigário, P. S. \& Costa, R. M. R. (2020). A COVID-19 e o distanciamento social: quando a onda da internet substituiu a onda do mar para a prática de exercícios físicos. Revista Augustus, 25(51), 335 356. https://doi.org/10.15202/1981896.2020v25n51p357

World Health Organization. (2020). Rolling updates on coronavirus disease (COVID-19) 2020. WHO; 2020. De https://www.who.int/emergencies/diseases/novel-coronavirus 2019/events-as-they-happen.

Wu, F., Zhao, S., Yu, B., Chen, Y. M., Wang, W., Song, Z. G., Hu, Y., Tao, Z. W., Tian, J. H., Pei, Y. Y., Yuan, M. L., Zhang, Y. L., Dai, F. H., Liu, Y., Wang, Q. M., Zheng, J. J., Xu, L., Holmes, E. C. \& Zhang, Y. Z. (2020). A new coronavirus associated with human respiratory disease in China. Nature. 579(7798):265-269. http://dx.doi.org/10.1038/s41586-020-2008-3. 